\title{
Evaluation of the dental structure loss produced during maintenance and replacement of occlusal amalgam restorations
}

Fernanda Sardenberg ${ }^{(a)}$ Clarissa Calil Bonifácio ${ }^{(b)}$ Mariana Minatel Braga ${ }^{(c)}$ José Carlos Pettorossi Imparato(d) Fausto Medeiros Mendes ${ }^{(d)}$

(a) MSc Student, Department of Pediatric Dentistry, School of Dentistry, Federal University of Minas Gerais, Belo Horizonte, MG, Brazil.

(b) MSc Student, Department of Restorative Dentistry, School of Dentistry, University of São Paulo, São Paulo, SP, Brazil.

(c) PhD Student; (d) Assistant Professors - Department of Pediatric Dentistry, School of Dentistry, University of São Paulo, São Paulo, SP, Brazil.

\section{Corresponding author:}

Clarissa Calil Bonifácio

Rua Santa Albina, 154

São Paulo - SP - Brazil

Cep: 05518-000

E-mail: clarissacalil@usp.br
Received for publication on Apr 12, 2007 Accepted for publication on Aug 02, 2007

\begin{abstract}
The aim of this in vitro study was to evaluate four different approaches to the decision of changing or not defective amalgam restorations in first primary molar teeth concerning the loss of dental structure. Ditched amalgam restorations $(\mathrm{n}=11)$ were submitted to four different treatments, as follows: Control group - polishing and finishing of the restorations were carried out; Amalgam group - the ditched amalgam restorations were replaced by new amalgam restorations; Composite resin group - the initial amalgam restorations were replaced by composite resin restorations; Flowable resin group - the ditching around the amalgam restorations was filled with flowable resin. Images of the sectioned teeth were made and the area of the cavities before and after the procedures was determined by image analysis software to assess structural loss. The data were submitted to ANOVA complemented by the Student Newman Keuls test $(\mathrm{p}<0.05)$. The cavities in all the groups presented significantly greater areas after the procedures. However, the amalgam group showed more substantial dental loss. The other three groups presented no statistically significant difference in dental structure loss after the re-treatments. Thus, replacing ditched amalgam restorations by other similar restorations resulted in a significant dental structure loss while maintaining them or replacing them by resin restorations did not result in significant loss.
\end{abstract}

Descriptors: Dentistry, operative; Dental amalgam; Dental restoration, permanent; Dental restoration failure. 


\section{Introduction}

The replacing of old restorations is still one of the procedures most frequently carried out by dentists in clinical practice. ${ }^{1,2}$ These replacements correspond to around two-thirds of all operative procedures carried out regularly, and this rate has not been reduced in spite of all the technological advancement in materials. ${ }^{3}$

One of the major challenges of current operative dentistry is the philosophy of Minimum Intervention. According to this philosophy, the repair of defective restorations is placed among other basic procedures, allowing the recovery of existing restorations, correcting possible imperfections and conserving what is conveniently restored, without sacrificing any remaining healthy dental structures. ${ }^{4}$

The decision criteria for replacing amalgam restorations are subjective and dentists often encounter amalgam restorations presenting secondary or recurrent caries, open contacts, body fracture, marginal fractures, poor contour/anatomic form, and overhangs. ${ }^{5}$ Replacing a restoration, however, does not guarantee that the same imperfections will not occur again, nor that new lesions and/or secondary caries lesions will not affect the new restoration. ${ }^{4}$

Maryniuk, Brunson ${ }^{6}$ (1989) believe that a marginal defect by itself is not a reason for replacing an amalgam restoration on the occlusal surface. Improvement in plaque control, diet, and the correct use of fluorides are indispensable for the reduction of that type of lesion, since occlusal amalgam restorations with ditching have similar characteristics to those of pit and fissure, and the essential in these cases is to control plaque accumulation. ${ }^{7}$ In addition, there is research evidence that secondary caries occurs more frequently in the gingival third of smooth aspects and proximal areas, demonstrating that a positive correlation between defective margins and secondary caries may not exist. ${ }^{8-10}$

Currently, there are other options for the effective control of amalgam restoration failure, namely the repair, the re-contouring and the sealing of the restoration. ${ }^{2,11}$ These alternatives allow the replacing of a restoration to be delayed, they minimize the wear of dental structures and involve less clinical time and money.
Considering the options above, it is valid to investigate the loss of dental structure associated to procedures of replacement and maintenance of amalgam restorations in primary molars. Thus, the aim of this in vitro study was to evaluate four different approaches to the decision of replacing or not amalgam restorations in primary molars concerning the loss of dental structure.

\section{Material and Methods}

For this study, forty-four human healthy primary first molars were chosen from the Human Tooth Bank, University of São Paulo (Ethics Committee approval n. 232/04). After being stored in saline solution at room temperature, the teeth were cleaned using a low-speed handpiece with pumice/water slurry and rinsed with tap water.

Each tooth was partially embedded in acrylic resin, using rectangular matrices. A class I occlusal cavity was made on each of them with a diamond bur number 3101 (KG Sorensen, São Paulo, SP, Brazil) using a water-cooled high-speed handpiece. Dimensions of the cavities were $2 \mathrm{~mm}$ in width (in the buccolingual direction), $4 \mathrm{~mm}$ in length (in the mesiodistal direction), and $1 \mathrm{~mm}$ in depth (as measured from the enamel-dentine junction). A millimeter ruler and a $\mathrm{K}$ file were used for standardization.

The restorations were made with encapsulated amalgam (Permite, SDI, Bayswater, Victoria, Australia). The procedures of insertion, condensation, burnishing and excess removal were carried out according to the manufacturer's instructions. The sculpture procedure was eliminated to standardize the sample.

After being stored for 24 hours in saline solution, one ditch around all amalgam restorations was made with round bur number 1011 (KG Sorensen, São Paulo, SP, Brasil) using a water-cooled high-speed handpiece. The diameter of the bur determined the ditch dimensions. All the procedures were carried out by the same operator.

The teeth were immersed in deionized water at $37^{\circ} \mathrm{C}$ for 24 hours and submitted to thermocycling - distilled water baths of 30 seconds, at $5^{\circ} \mathrm{C}( \pm 2)$ and $55^{\circ} \mathrm{C}( \pm 2)$, totalizing 700 cycles. ${ }^{12}$ After that, the teeth were sectioned at the centre of the restora- 
tions (in the buccolingual direction) using a $0.3 \mathrm{~mm}$ thick diamond saw mounted on a microtome (Labcut 1010, Extec Co., Enfield, CT, USA).

The specimens were analyzed with a stereomicroscope at a $10 \mathrm{X}$ magnification and reflected light (SZPT Olympus, Tokyo, Japan), and the images of the sectioned teeth were stored in a computer. The areas of the teeth were determined by an image analysis software (Leica Qwin, Leica Microsystems, Heidelberg, Germany) on each side of the sections (in $\mathrm{mm}^{2}$ ). After that, the specimens were joined using rectangular matrices and the teeth were randomly divided into four groups $(\mathrm{n}=11)$.

- Control group (CT): burnishing and finishing of the amalgam restorations were performed using a special bur to finish the amalgam restorations, under low-speed.

- Amalgam group (AM): removal of the amalgam restorations was performed with a round bur number 1011 (KG Sorensen, São Paulo, Brazil) under water-cooling and high-speed, followed by the making of a new cavity for amalgam, extending up to the ditch and the placing of a new restoration using amalgam (Permite, SDI, São Paulo, Brazil).

- Resin group (RS): removal of the amalgam restorations was performed with a round bur number 1011 (KG Sorensen, São Paulo, Brazil) under water-cooling and high-speed, followed by the placing of new restorations in micro hybrid composite resin (Universal Amelogen - Ultradent, UT, USA), also including the ditching; the micro hybrid composite was inserted with the needle provided by the manufacturer into the cavities using an incremental technique, and each increment was light-cured for $30 \mathrm{~s}$.

- Flowable composite resin group (FR): finishing and burnishing of the amalgam restorations were carried out with the same bur of CT, followed by the fulfilling of the ditching with flowable composite resin (Tetric Flow - Vivadent, Schaan, Liechtenstein), inserted with the needle provided by the manufacturer.

After the treatments, the teeth were re-submitted to thermocycling and re-sectioned. New images were made at the same position as previously described and the teeth were measured again by the same examiner using the same software. The loss of dental structure in each one of the studied groups was determined by subtracting the area of the teeth before the procedure from the area after the procedures.

The Kolmogorov-Smirnov test was used to evaluate if the samples presented normal distribution. As the values were normally distributed, we used the paired Student's $t$ test to compare the area of each tooth before and after the procedures in each group. The loss of dental structure was evaluated by subtracting the tooth areas before and after the procedures from each other and, after that, by comparing the obtained differences among the different groups, using ANOVA complemented by the Student Newman Keuls test. The level of significance was set at $\mathrm{p}<0.05$.

\section{Results}

In all groups, significant differences were observed between the areas of the teeth before and after the treatments, indicating loss of dental structure (Figure 1).

The means of the differences between the measures of the teeth areas before and after the treatments are expressed in Graph 1. The difference observed in AM was significantly higher than that of the other groups (Graph 2). The other three groups did not present statistically significant differences concerning the loss of dental structure.

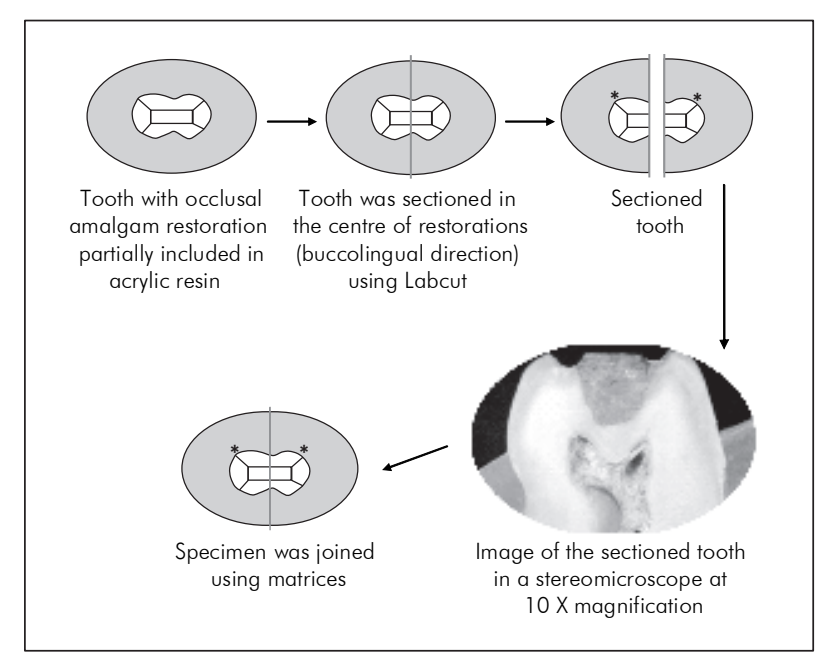

Figure 1 - Schematic representation of the procedures. 


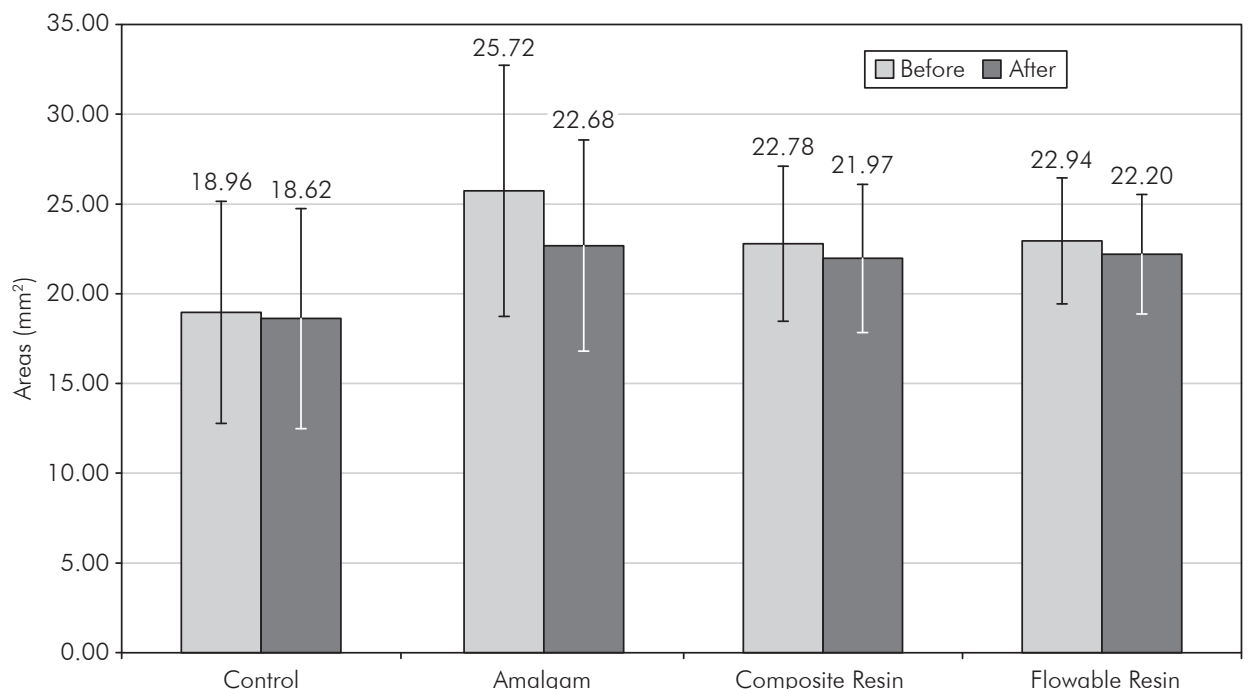

Graph 1 - Teeth areas before and after the treatments (mean and standard deviation vertical lines).

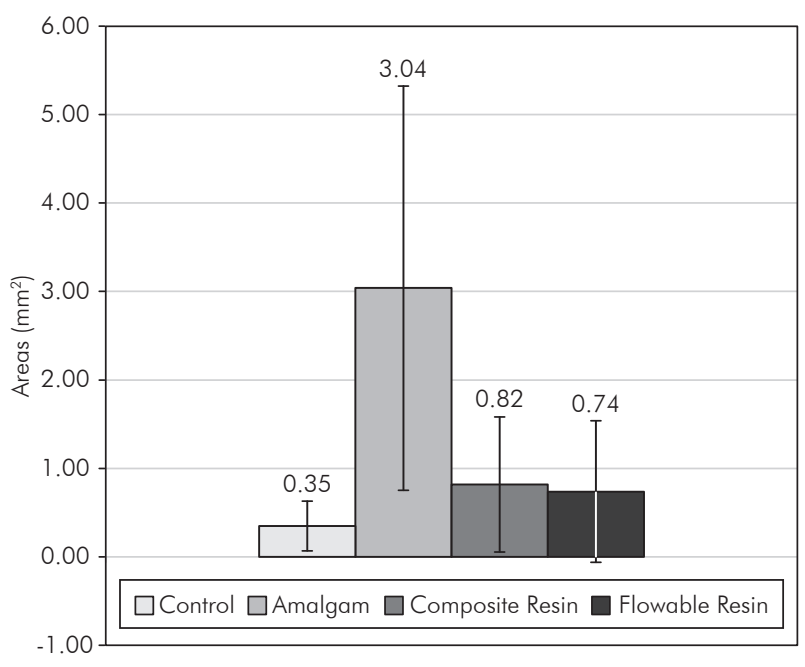

Graph 2 - Differences between the means of the teeth areas. The vertical lines indicate the Standard Deviation.

\section{Discussion}

Currently, the procedures involving the replacement of restorations have exceeded the procedures involved in the restoration of new lesions. ${ }^{1}$ However, the replacement of restorations tends to cause loss of healthy dental structure ${ }^{1}$ leading to a repeated restoration cycle. ${ }^{13}$

Re-restoring teeth is an important component of operative dentistry, and the perceived presence of secondary caries is a major reason for undertaking it. In the absence of a diagnosis of secondary caries, a morphologic discrepancy at the margin of a restoration commonly provides the necessary justification for the replacement. ${ }^{13}$ The ditching in restorations by itself should not be, however, the unique criterion for replacing restorations. ${ }^{3}$ This imperfection may be addressed by repairing or re-contouring the restoration ${ }^{2}$, thus not requiring the replacement of the restoration. ${ }^{11}$

Although alternative options to the replacement of restorations are available, there is no consensus among dentists about which kind of procedure should be adopted and in which circumstances. ${ }^{2}$ For those reasons, this study had the objective of evaluating different procedures for the repair of amalgam restorations with marginal defects, concerning the loss of sound dental structure.

Loss of dental structure was observed in all the groups. Nonetheless, in those groups in which the restoration was maintained (CT and FR) or the amalgam restoration was replaced by a resin restoration (RS), the loss of sound tissue was smaller (less than $1 \mathrm{~mm}^{2}$ ). It can be inferred that the loss of dental structure is a consequence inherent to any treatment. Therefore, dentists must avoid replacing restorations for no reason. This choice must be limited to the cases where secondary caries is present.

The highest loss of healthy dental structure was observed in the group where the amalgam restoration with ditching was replaced by another amalgam restoration. This restoring material requires the preparation of a retentive cavity, following the proper operative principles, leading to greater tooth wear since amalgam does not have adhesion to den- 
tal tissues and its retention is obtained by the design of the cavity in which it is inserted..$^{14}$ Differently, the retention of composite resin is based on adhesion of the adhesive system to the dental structure. ${ }^{15}$

Dental restorative treatment plays a supporting role in the philosophy of health promotion, whose goals are maximum prevention and minimal intervention. The maintenance of a restoration or the sealing of its margins must be considered as treatment options since they are approaches that involve minor loss of dental structure and agree with the philosophy of health promotion. The procedures of finishing and burnishing or margin sealing restore dental anatomy, eliminate clinical steps and reduce the consuming of healthy dental structure, increasing the useful life of a restoration and minimizing the necessity of replacement. ${ }^{3,16-19}$

\section{References}

1. Adegbembo AO, Watson PA. Removal, replacement and placement of amalgam restorations by Ontario dentists in 2002. J Can Dent Assoc. 2005;71(8):565.

2. Setcos JC, Khosravi R, Wilson NH, Shen C, Yang M, Mjor IA. Repair or replacement of amalgam restorations: decisions at a USA and a UK dental school. Oper Dent. 2004;29(4):392-7.

3. Anusavice KJ. Criteria for placement and replacement of dental restorations. Fla Dent J. 1988;59(2):30-1.

4. Elderton RJ. Overtreatment with restorative dentistry: when to intervene? Int Dent J. 1993;43(1):17-24.

5. Penning C. Repair and revision 1. Repair or replacement of amalgam. Ned Tijdschr Tandheelkd. 2001;108(2):46-9.

6. Maryniuk GA, Brunson WD. When to replace faulty-margin amalgam restorations: a pilot study. Gen Dent. 1989;37(6):4637.

7. Pimenta LA, Navarro MF, Consolaro A. Secondary caries around amalgam restorations. J Prosthet Dent. 1995;74(3):21922.

8. Kidd EAM, O'Hara JW. The caries status of occlusal amalgam restorations with marginal defects. J Dent Res. 1990;69(6):1275-7.

9. Mjor IA. Repair versus replacement of failed restorations. Int Dent J. 1993;43(5):466-72.

10. Soderholm K, Antonson DE, Fischelsschwerger W. Correlation between marginal discrepancies at the amalgam/tooth interface and recurrent caries. In: Anusavice KJ. Quality evaluation of dental restorations / International Symposium on Criteria for Placement and Replacement of Dental Restorations. Lake Buena Vista, Florida, October 19-21, 1987. Chicago: Quintessence Book; 1989. p. 97-107.
The repair of old amalgam restorations in permanent teeth has shown clinical success rates similar to those obtained by replacing them after periods of less than 5 years. ${ }^{11,20}$ On the other hand, after 10 years, repaired amalgam restorations have shown higher indexes of imperfection than those of remade ones. ${ }^{20}$ For primary teeth, however, clinical studies that might corroborate the findings of this in vitro study are required in order to prove the effectiveness of the techniques that cause less loss of dental structure.

\section{Conclusion}

In conclusion, all the studied procedures involved loss of healthy dental structure, but the replacing of an old amalgam restoration by a new one made of the same material entailed greater structural loss and should thus be avoided.

11. Gordan VV, Riley JL $3^{\text {rd }}$, Blaser PK, Mjor IA. 2-year clinical evaluation of alternative treatments to replacement of defective amalgam restorations. Oper Dent. 2006;31(4):418-25.

12. Crim GA, Garcia-Godoy F. Microleakage: the effect of storage and cycling duration. J Prosthet Dent. 1987;57(5):574-6.

13. Elderton RJ. Clinical studies concerning re-restoration of teeth. Adv Dent Res. 1990;4:4-9.

14. Goldfogel MH, Smith GE, Bronberg TJ. Amalgam polishing. Oper Dent. 1976;1(4):146-50.

15. Baratieri LN, Ritter AV. Four-year clinical evaluation of posterior resin-based composite restorations placed using the total-etch technique. J Esthet Restor Dent. 2001;13(1):50-7.

16. Boyd MA, Richardson AS. Frequency of amalgam replacement in general dental practice. J Can Dent Assoc. 1985;51(10):7636.

17. Cardoso M, Baratieri LN, Ritter AV. The effect of finishing and polishing on the decision to replace existing amalgam restorations. Quintessence Int. 1999;30(6):413-8.

18. Cipriano TM, Santos JF. Clinical behavior of repaired amalgam restorations: a two-year study. J Prosthet Dent. $1995 ; 73(1): 8-11$.

19. Oleinisky JC, Baratieri LN, Ritter AV, Felipe LA, de Freitas SF. Influence of finishing and polishing procedures in the decision to replace old amalgam restorations - An in vitro study. Quintessence Int. 1996;27(12):833-40.

20. Smales RJ, Hawthorne WS. Long-term survival of repaired amalgams, recemented crowns and gold castings. Oper Dent. 2004;29(3):249-53. 